Revue des patrimoines

\title{
Naissance d'un patrimoine : les projets de classement des ruines, vestiges et souvenirs de guerre (1915-1918)
}

\section{Franck Viltart}

\section{OpenEdition}

\section{Journals}

Édition électronique

URL : http://journals.openedition.org/insitu/10990

DOI : 10.4000/insitu. 10990

ISSN : 1630-7305

Éditeur

Ministère de la culture

Référence électronique

Franck Viltart, " Naissance d'un patrimoine : les projets de classement des ruines, vestiges et souvenirs de guerre (1915-1918)», In Situ [En ligne], 23 | 2014, mis en ligne le 04 mars 2014, consulté le 19 avril 2019. URL : http://journals.openedition.org/insitu/10990 ; DOI : 10.4000/insitu.10990

Ce document a été généré automatiquement le 19 avril 2019

\section{(c)}

In Situ Revues des patrimoines est mis à disposition selon les termes de la licence Creative Commons Attribution - Pas d'Utilisation Commerciale - Pas de Modification 4.0 International. 


\title{
Naissance d'un patrimoine : les projets de classement des ruines, vestiges et souvenirs de guerre (1915-1918)
}

\author{
Franck Viltart
}

1 De nombreux monuments et sites de la Première Guerre mondiale sont aujourd'hui inscrits ou classés au titre des monuments historiques. Si à l'aube du centenaire de cette guerre, la conservation de certains lieux de mémoire continue de faire débat, il semble important de revenir sur un processus de patrimonialisation qui prend ses racines durant la guerre elle-même.

2 En 1915, alors que les bombardements mettent à terre une grand partie du patrimoine architectural le long de la ligne de front, plusieurs propositions émergent en France afin de classer certains vestiges au nom de l'histoire et de la mémoire nationale. Très vite, à côté des ruines et des monuments endommagés, ce processus fait émerger un patrimoine de guerre fait d'installations militaires, d'abris et de tranchées. L'idée de protéger les traces d'une guerre bousculait les principes tout juste promulgués par la loi de 1913 sur les monuments historiques. Il s'agissait alors de conférer aux vestiges un statut qui permettrait leur conservation pour les générations futures. Ruines et vestiges mais aussi champs de bataille, à partir de quels critères a-t-on voulu classer ces éléments et surtout quel sens a-t-on voulu donner à cet effort de patrimonialisation sans précédent?

\section{Les « ruines historiques »}

3 Les destructions de l'année 1914 provoquent une vague d'émotion dans l'opinion publique en France et en Belgique, suscitée notamment par la mise en avant de villes-martyres comme Ypres, Reims ou Soissons. Face aux destructions de certains monuments, une question se pose très vite: reconstruire ou conserver les ruines? L'idée que les monuments devaient renaître des ruines s'oppose assez tôt à une volonté chez certains de conserver un certain nombre de vestiges, témoignages édifiants du conflit ${ }^{1}$. En 1915, la 
commission de Réparation des dommages de guerre, en visite à Arras, notait ainsi dans son rapport : «Peut-être serait-il bon [...] de choisir quelques-unes de ces ruines et de les garder intactes pour les générations futures. Elles feront mieux comprendre ce qu'est la guerre et ne permettront pas même de pardonner $»^{2}$.

L'administration des Monuments historiques ne tarde pas à s'intéresser à la question des ruines et lance au printemps 1915 une inspection pour mesurer l'ampleur des destructions. L'inspecteur général des Monuments historiques, Julien Berr de Turique, reçoit pour mission de visiter les monuments détruits près de la ligne de front en Picardie et dans le Nord, au mois de juillet $1915^{3}$.

Une telle démarche rencontrait certaines motivations plus politiques. Conserver les traces des destructions permettait de mettre en scène des preuves de «la barbarie allemande » sur le territoire français. C'est face à ces enjeux que le 23 septembre 1915, le député du Nord Jules-Louis Le Breton déposa une proposition de loi ouvrant des possibilités de classement et de conservation des ruines produites par les opérations de guerre (fig. $\mathbf{n}^{\circ} \mathbf{1}$ ). La proposition de loi commençait ainsi :

Partout sur notre front, l'obus et l'incendie ont accumulé les ruines, amoncelé les décombres. Nombre de ces ruines sont pleines de souvenirs militaires et constituent des pages singulièrement émouvantes et tragiques d'une des périodes les plus belles de notre histoire nationale; on y lit, mieux que dans l'ouvrage du plus puissant historien, la vaillance admirable de nos soldats et la sauvage barbarie des troupes allemandes ${ }^{4}$.

\section{Figure 1}

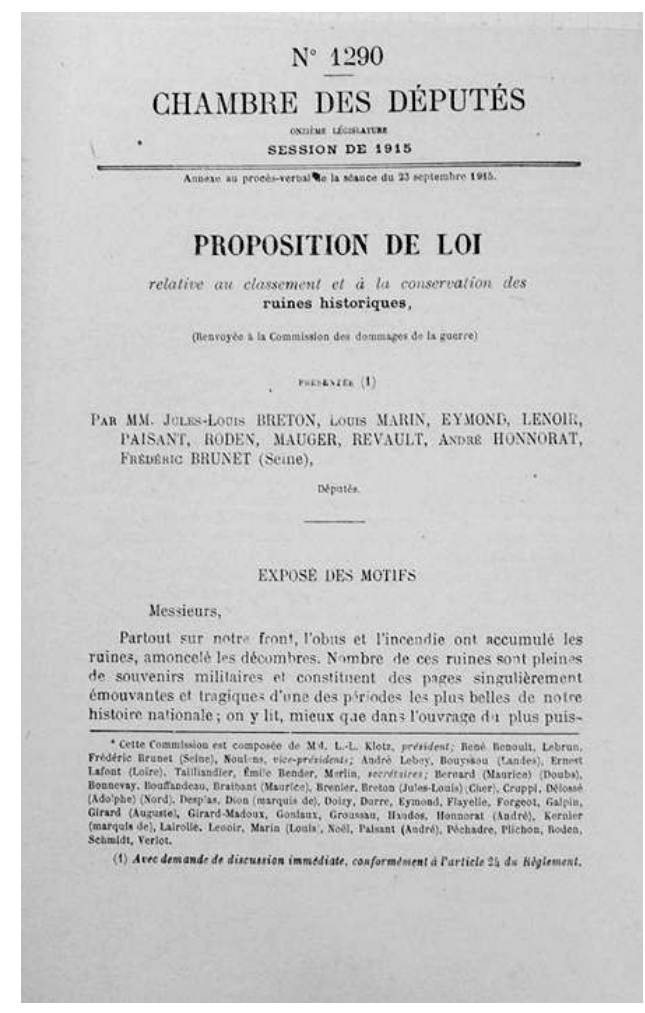

Proposition de loi relative au classement et à la conservation des ruines historiques, présentée par Jules-Louis Le Breton, 23 septembre 1915. MAP 80/03/36.

(c) Médiathèque de l'architecture et du patrimoine. 
6 Évoquant au présent les bombardements allemands, sur Ypres notamment, le député souhaite que soit conféré dès à présent un statut particulier aux ruines qu'il convient, selon lui, de conserver d'urgence pour leur « entière vérité historique ». Ainsi, précise-til, «il faut que nous léguions intacts quelques-uns de ces souvenirs tragiques aux générations futures ", témoignages " de la grande période que nous vivons ", rejoignant ici l'idée d'une Grande Guerre vécue dès 1915. Les enjeux de ce texte sont évidents, puisqu'il y souligne l'usage que l'on pourra faire de ces ruines, servir une mémoire nationale à la gloire «du sang des généraux de nos héroïques soldats » victimes du « militarisme allemand ». Les ruines serviraient ici d'illustration à un récit héroïque de la guerre.

7 En matière de protection, le projet de loi repose sur le classement au titre des monuments historiques des ruines, mais n'hésite pas à proposer également des forêts et des champs de bataille entiers. Argument à destination de l'administration des Monuments historiques, le député avance qu'il ne faudrait pas laisser se détruire ces vestiges comme l'ont été au XIX ${ }^{e}$ siècle certains vestiges du Moyen Âge. Il fait appel pour cela aux nouveaux outils fournis par la loi de 1913, et propose de mettre en place à ce titre des mesures d'expropriation. Il n'hésite pas à mettre en avant «l'intérêt général » à conserver certaines ruines. Utilisant la loi de 1913, il propose que sur des terrains privés, les propriétaires de certains vertiges puissent être expropriés. Il y ajoute cependant la possibilité du versement d'une indemnité de la part de l'État. Une proposition qui est peu appréciée par le ministre des Finances qui, dans une note adressée au président du Conseil ${ }^{5}$, met en garde contre la «stérilisation » qui pourrait découler de telles mesures, avec des centres urbains ainsi conservés à l'état de ruine. Il insiste également sur les dangers de l'expropriation des populations et surtout le rachat de biens qui auraient évidemment perdu leur valeur d'avant-guerre. Un avis qui est partagé par le soussecrétaire d'État en charge des Beaux-Arts, Albert Dalimier ${ }^{6}$. Ce dernier reste cependant attaché à l'idée selon laquelle il appartient au service des Monuments historiques d'envisager de classer certaines ruines qui, selon lui, seront irrémédiablement visitées après la guerre. Si le projet de loi Le Breton n'aboutit pas, il convainc de l'intérêt porté aux vestiges de guerre, non seulement par l'administration mais également par d'autres acteurs, comme ceux du tourisme.

\section{Des lieux de pèlerinage et de tourisme}

Le projet de loi Le Breton envisageait de donner aux ruines une dimension mémorielle et pédagogique, puisqu'il fallait, selon le député, «que demain et pour toujours, ces ruines historiques deviennent un lieu de pèlerinage patriotique où seront conduits les enfants de nos écoles ». Il fallait s'assurer aussi, d'après ce texte, que les anciens combattants puissent après-guerre "aller revoir leurs tranchées ", ouvrant la voie dès 1915 aux nombreux pèlerinages patriotiques, les traces du conflit permettant au combattant de rassembler ses souvenirs. Bien plus étonnant, le texte aborde directement l'intérêt économique du classement des ruines à travers sa dimension touristique. Selon le projet de loi de 1915, une ville détruite pourrait être reconstruite non pas sur son emplacement originel, mais à proximité : ainsi, «la vieille cité morte devenant pour la cité nouvelle édifiée à ses côtés une source de richesses en attirant les touristes et les visiteurs ». 
9 Le tourisme de guerre ne naît pas avec la Première Guerre mondiale : c'est au lendemain de la guerre de Sécession qu'ont été publiés les premiers guides des champs de bataille et organisés les pèlerinages d'anciens combattants ${ }^{7}$. En revanche, les pratiques touristiques déjà bien implantées dans une partie de la société française en 1914 vont largement participer à la protection des sites de la Grande Guerre. À la veille de 1914, les acteurs du tourisme jouaient déjà un rôle significatif dans le domaine patrimonial, selon Jean-Michel Leniaud, pour qui :

La fin du XIX siècle n'a pas seulement vu l'entrée des intellectuels et des artistes sur la scène de la restauration monumentale. La revendication d'appropriation collective que ce phénomène exprime s'accompagne d'une tendance à laquelle se trouve plus ou moins lié le développement touristique ${ }^{8}$.

10 Acteur principal du développement du tourisme, le Touring Club de France, avec ses 100000 membres en 1906, achève en 1914 les 34 volumes de sa revue Sites et monuments. Cette association écrit au sous-secrétaire d'État des Beaux-Arts en 1915, afin de l'informer qu'il y aurait un certain intérêt à conserver une partie des sites de la guerre en cours. Le lobbying qu'opère le Touring Club de France se manifeste à plusieurs reprises. Le 5 juin 1916, le président du comité des Sites et monuments au sein de l'association informe Paul Léon, chef du service de l'architecture au sous-secrétariat aux Beaux-Arts, de la nécessité de maintenir à l'état de ruine certains édifices ravagés. Il affirme que son comité a relevé plusieurs sites intéressants et que notamment, « son attention s'est également portée sur certains éléments de tranchées de Champagne particulièrement intéressants par leurs dispositions intérieures ou la décoration qu'ils ont reçue de l'ingéniosité ou du goût artistique de nos soldats ${ }^{9} »$.

11 Au milieu des batailles de l'année 1915 et alors que des milliers d'hommes mouraient dans les tranchées, le tourisme de guerre ne pouvait se mettre en place sans un certain contrôle des autorités publiques. Le 18 octobre 1916, l'office national du Tourisme, dépendant du ministère des Travaux Publics, s'empare de la question par une délibération qui inquiète le sous-secrétaire aux Beaux-Arts, Albert Dalimier. Si ce dernier est convaincu de la protection qu'il faut désormais apporter à certaines ruines, il souhaite que l'on interdise leur utilisation à des fins commerciales. Dans une lettre au garde des sceaux $^{10}$, il met en garde contre le commerce des reliques ou à la marchandisation de quelconques droits de visite de la part de propriétaires privés. Il en va de la responsabilité de l'État, selon lui, car «il y a des immeubles témoins de tant d'héroïsme et de tant de sacrifices, des lieux de pèlerinage et dont il appartient à l'État d'assurer la conservation, en empêchant qu'ils ne soient profanés par certains usages commerciaux ». Il va jusqu'à ironiser : « Comprendrait-on qu'il fût permis d'ouvrir un débit de vins ou une guinguette à la ferme Navarin [...] ? ».

12 On sait que les enjeux touristiques autour des sites de guerre ont été pris au sérieux par l'administration et notamment en ce qui concernait le transport des visiteurs. Le 2 mars 1917, l'office national du Tourisme prévoyait même de proposer de légiférer sur le logement des visiteurs pour les trois années qui suivraient la cessation des hostilités dans la zone des armées ${ }^{11}$. En classant certains vestiges, l'administration devait également s'assurer du fait que les moindres droits de visite reviendraient à l'État. Il aurait été en effet très délicat pour l'État de voir proliférer des entrepreneurs privés réclamant un droit de visite sur des sites où reposaient encore des corps de soldats français.

13 Sur les territoires du front bouleversés par la guerre, ces projets de classement reçoivent une attention particulière. Le 16 avril 1917, alors que le général Nivelle lance sa grande 
offensive au Chemin des Dames dans l'Aisne, le conseil général de la Meurthe-et-Moselle, qui abrite provisoirement la commission des Sites de la Meuse, propose d'inclure des lieux de combats dans les sites retenus pour le classement, modifiant ainsi les dispositions de la loi de 1906. Le conseil général du Pas-de-Calais se prononce de la même façon pour le classement de ses sites de guerre, le 25 septembre $1917^{12}$, ouvrant la porte à toute mesure de classement. L'idée d'un tourisme de guerre et la volonté de certains départements de classer certains sites rendaient encore plus nécessaires l'inventaire et la protection des vestiges de guerre.

\section{La création de la commission des Vestiges et souvenirs de guerre}

Le projet de loi de 1915 proposait la création d'une « commission des Ruines historiques », qui ne voit pas le jour malgré une première proposition de composition présentée par le sous-secrétaire d'État Albert Dalimier, qui plaçait Paul Léon à sa tête ${ }^{13}$. Les événements militaires allaient donner l'occasion à l'administration de donner naissance à la première instance de classement des vestiges de guerre.

En mars 1917, le retrait des troupes allemandes d'une partie du front entre Arras et Soissons, conformément au plan Alberich, libère un grand nombre de communes françaises. Le service de "Protection des œuvres artistiques dans la zone des armées » constate qu'une grande partie des monuments classés avant-guerre dans ces communes sont à terre. En avril, dans une lettre au ministre de la Guerre, le sous-secrétaire d'État aux Beaux-Arts propose, selon le vœu du comité interministériel chargé d'étudier les mesures de reconstruction des pays dévastés, la création d'une commission des "Souvenirs et vestiges de guerre ${ }^{14}$. Cette commission sera chargée d'inventorier et d'étudier les vestiges mais également de proposer certains d'entre eux au classement.

Mise sur pied en mai 1917, la commission rassemble des représentants des ministères de la Guerre, des Beaux-Arts, des Travaux Publics et du ministère de Régions libérées. Paul Léon, pour les Monuments historiques et Georges Famechon, qui préside l'office national du Tourisme, sont conviés. La commission se fixe comme premier objectif de « reconnaître les terrains et certaines portions des anciens fronts qui méritent d'être conservés dans l'état où ils se trouvent, soit pour le souvenir des faits d'armes dont ils on été le théâtre, soit en raison de leur aspect ou de leur organisation particulièrement typique $»^{15}$. Elle confie pour cela à l'architecte en chef des Monuments historiques André Ventre une mission d'inspection et d'inventaire. Il doit être accompagné par le commandant Viel de l'État-major de l'armée et du chef de la Section photographique de l'armée, Pierre Marcel, opérateur expérimenté aux prises de vue sur le front.

17 La commission des Vestiges et souvenirs de guerre est attachée lors de sa création en 1917 au ministère de la Guerre et dépend donc du pouvoir militaire. La commission demeure d'ailleurs présidée dans un premier temps par un officier supérieur. La présidence échoue au général Vidalon, puis à partir de septembre 1917, au général Savatier. Les choses sont prises très au sérieux par le Grand Quartier général (GQG), où le général Debeney demande de faire établir des listes de terrains ou de localités susceptibles de contenir des vestiges intéressants et de prendre les mesures conservatoires sur ces terrains en attendant l'intervention et la décision de la commission ${ }^{16}$. 
Nommé architecte en chef des Monuments historiques en Haute-Marne en 1915, André Ventre venait d'être installé dans la Meuse en 1917. Dans ces régions du front, il avait entrepris lui-même de nombreux relevés de certains exemples de l'architecture rurale traditionnelle dans la perspective de la reconstruction ${ }^{17}$. Aux prises avec les problèmes des destructions et doté d'un talent de dessinateur, c'est donc naturellement à lui que la commission des Vestiges et souvenirs de guerre confie le soin d'établir des plans et des croquis de ces vestiges à l'occasion des premières inspections qui débutent au mois de juin 1917 dans la zone évacuée par les Allemands en Picardie.

\section{Inspections, inventaire et premières propositions de classement}

19 Du 16 au 19 juin 1917, André Ventre et le commandant Viel mènent une première visite sur l'ancienne ligne de front, depuis le village de Nouvron-Vingré à l'est de Soissons dans l'Aisne, jusqu'à Tilloloy dans la Somme, en passant par le Noyonnais dans l'Oise. La visite est guidée par le capitaine Garcin de la $3^{\mathrm{e}}$ armée, qui est mis à disposition par le GQG. Cette première inspection donne l'occasion à André Ventre d'établir les critères du classement qu'il souhaite proposer à la commission. Un rapport est rédigé le $1^{\mathrm{er}}$ juillet $1917^{18}$. André Ventre s'y montre très soucieux d'opérer un tri parmi les vestiges rencontrés. Dans ses remarques préalables, il propose de distinguer deux catégories :

1. les ensembles, constitués de villages en ruine et de mouvements de terrains organisés défensivement,

2. les vestiges isolés, poste de commandement, abris, etc.

Notant l'aspect beaucoup plus intéressant des ensembles par rapport aux vestiges isolés, tant au point de vue historique que pédagogique, l'architecte déploie un argumentaire pour chaque site proposé au classement. Ainsi, le tout premier site visité par les deux délégués, le village de Nouvron-Vingré, est entièrement à terre. Les deux délégués proposent de le ranger parmi les ensembles intéressants mais l'excluent de tout classement car, selon eux : «il faut permettre au village de renaître ». Les deux délégués proposent de conserver l'aspect des ruines uniquement par la constitution d'une documentation photographique et de dessins. Ils notent cependant que les galeries creusées par les Allemands sur le plateau pourraient être classées sans que cela nuise à la reprise économique du village. Ils visitent ensuite l'ancienne ferme de Confrécourt, bien que ce site n'ait pas été proposé par le GQG. Ils proposent de ne pas classer la ferme médiévale qui, selon eux, « renaîtra de ses ruines ». Ils souhaitent cependant proposer au classement la tour de la ferme et sa tourelle blindée, encore en place en 1917 (fig. $\mathbf{n}^{\circ} \mathbf{2}$ ). Les anciennes carrières de pierre qui se trouvent en contrebas de la ferme ne semblent pas les intéresser, ils les qualifient d'abris "qui ne présentent pas un intérêt capital ", malgré les innombrables gravures et autels sculptés qui s'y trouvent ${ }^{19}$. Le petit cimetière provisoire français dans la pente semble retenir un peu plus leur attention, puisqu'ils l'inscrivent sur la liste des «vestiges à conserver». Un peu plus loin, le poste de commandement de Chapeaumont, plus connu sous le nom de PC Reboul, est rangé parmi les « vestiges isolés à classer ». Au même titre, le curieux arbre-observatoire près de Bitry sera, selon eux, facile à conserver (fig. $\left.\mathbf{n}^{\circ} \mathbf{3}\right)\left(\right.$ (fig. $\left.\mathbf{n}^{\circ} \mathbf{4}\right)$. 
Figure 2

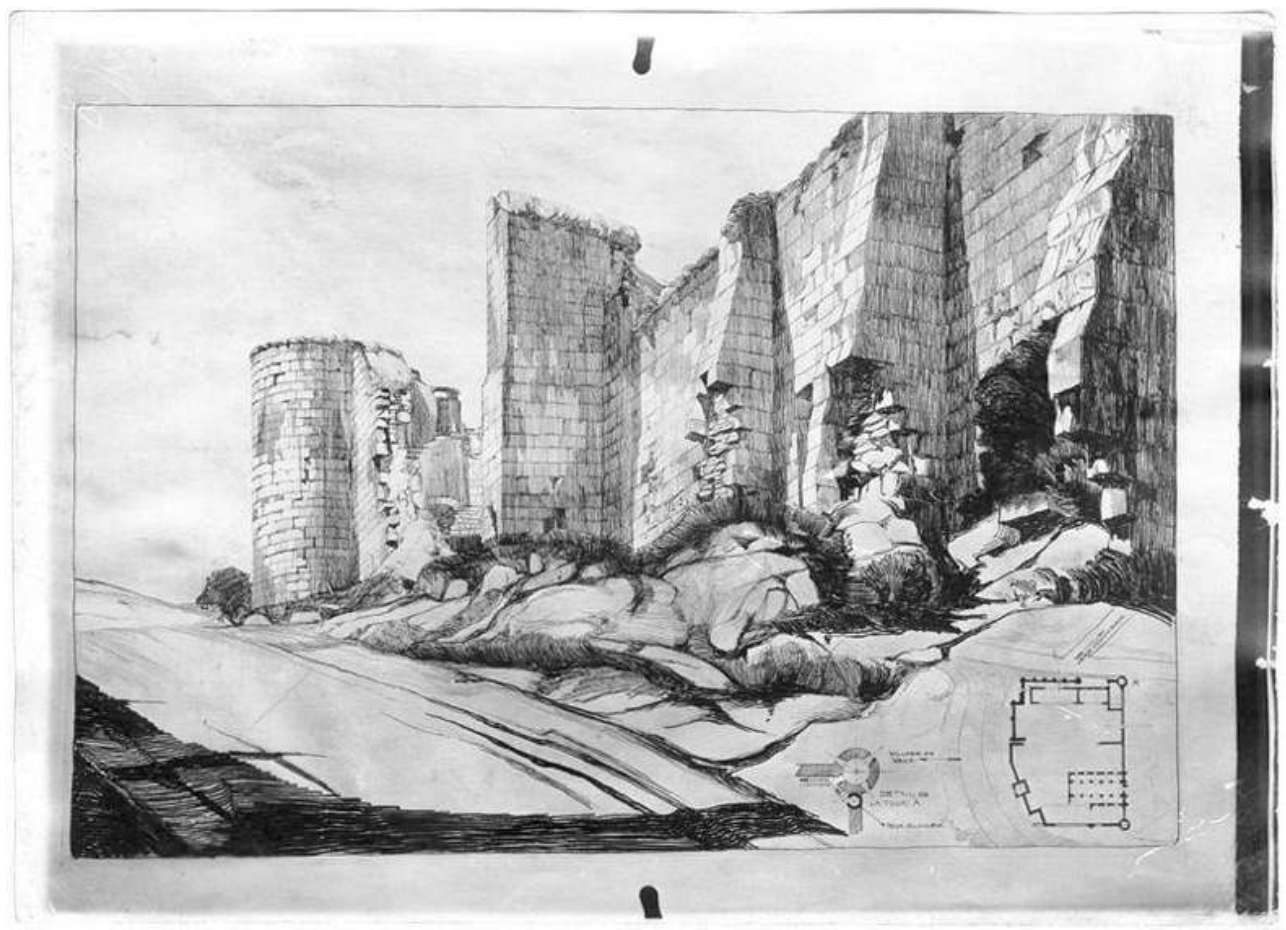

La ferme de Confrécourt (Aisne), détail de la tour, dessin d'André Ventre, juin 1917. (c) Médiathèque de l'architecture et du patrimoine.

\section{Figure 3}

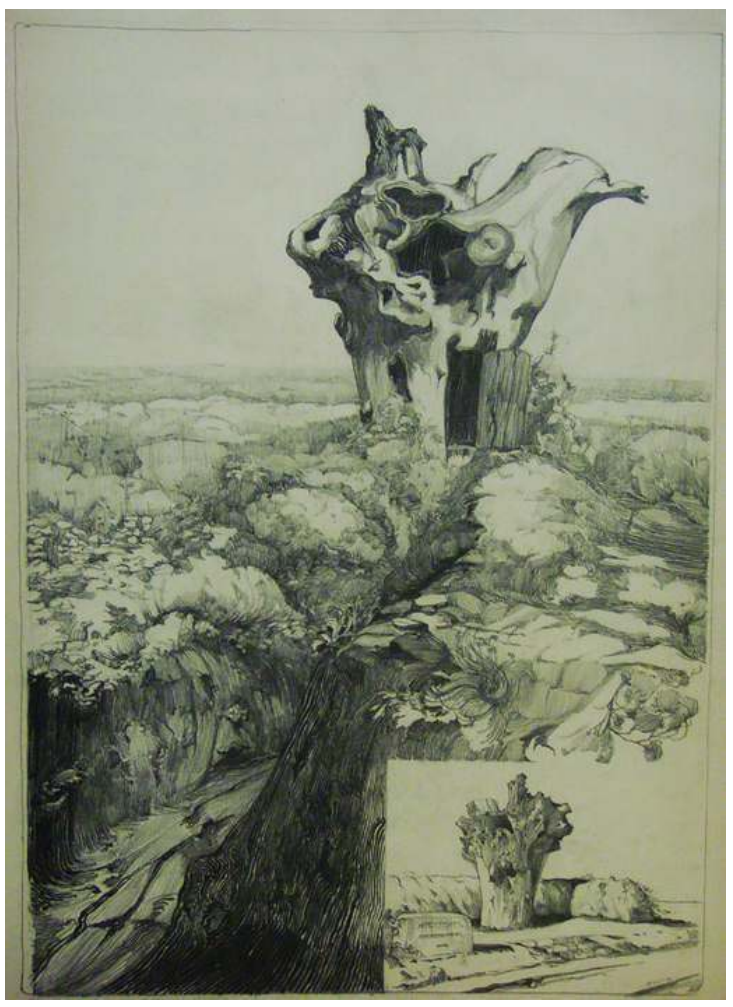

Arbre-observatoire de Bitry (Oise), dessin d'André Ventre, juin 1917 (non coté)

Phot. Viltart, Franck. (c) Médiathèque de l'architecture et du patrimoine. 
Figure 4

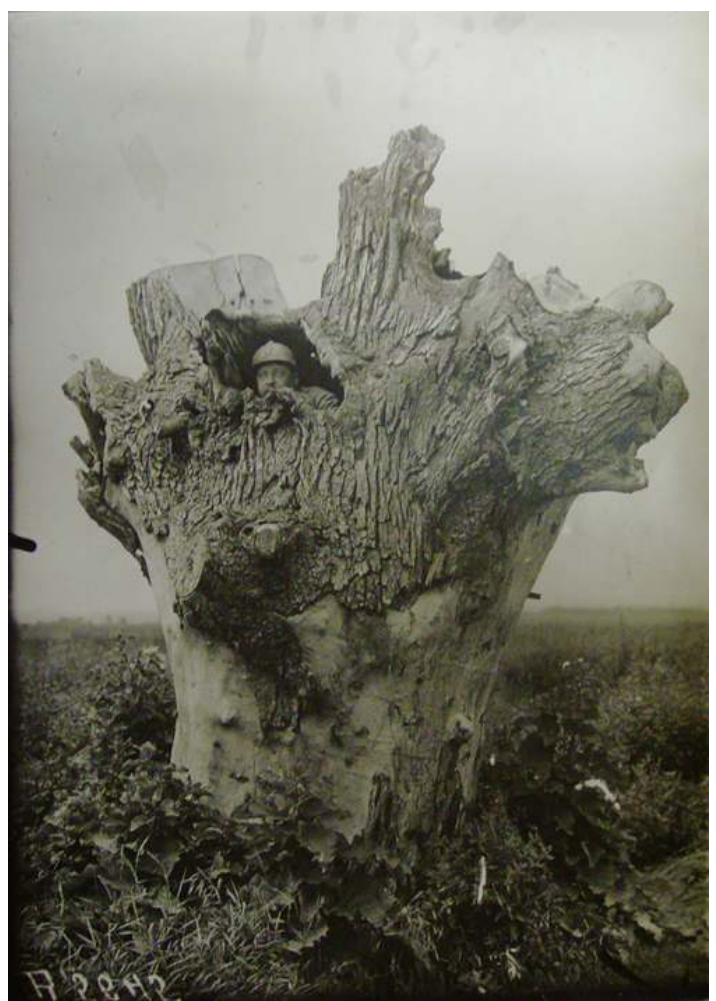

Arbre-observatoire de Bitry (Oise), photographie, juin 1917 (non coté).

(C) Médiathèque de l'architecture et du patrimoine.

Non loin de là, l'abri des officiers allemands dans le village de Nampcel, épargné lors du retrait allemand, retient aussi leur attention. Bien que plusieurs abris d'officiers subsistent à divers endroits du front, celui-ci mérite selon eux d'être classé, "par son importance, par le fini et le définitif de sa construction ». À Moulin-sous-Touvent, ils notent qu'il « est à remarquer que toutes les carrières de la région ont été aménagées par les Allemands en abris souterrains ; on ne saurait songer à les classer toutes ", précisentils. Ils relèvent cependant la présence de très nombreux blasons et emblèmes qui montrent que les carrières ont été occupées par un grand nombre d'unités. Ils proposent de classer l'entrée de ces carrières, car le reste pourrait être encore exploité et serait de toute façon trop dangereux à visiter. Ils proposent ici, singulièrement, de mettre des panneaux en cas de classement où il serait précisé que l'administration décline toute responsabilité en cas d'accident.

En continuant sur le plateau en direction de Noyon, ils croisent la ferme de Quennevières, qui pourra être classée, selon eux, en souvenir de l'offensive qui s'y déroula en juin 1915, témoin de l'héroïsme des zouaves. Les délégués vont même jusqu'à proposer dans leur rapport le rachat par l'État du site. Les délégués sont particulièrement impressionnés par le site du cimetière communal de Tracy-le-Val, entièrement organisé par les Allemands, avec des abris, tranchées et tunnels (fig. $\mathbf{n}^{\circ} 5$ ). Ils rangent cet ensemble dans les organisations «classables" pour la bonne raison qu'il sera impossible de rendre à cet endroit son usage originel. Ils visitent ensuite un ensemble d'installations allemandes à Chiry-Ourscamp, avec un abri pour officiers, des abris pour la troupe, ainsi que plusieurs postes d'observation et d'artillerie, qu'ils souhaitent faire photographier mais dont seul 
l'abri des officiers pourrait être classé (fig. $\mathbf{n}^{\circ} \mathbf{6}$ ). Bien que significative à leurs yeux, il appartiendra à la commission de donner son avis sur le classement de la Kommandantur aménagée dans une maison du centre du village, intéressant symbole de « la puissance allemande » selon les délégués.

Figure 5

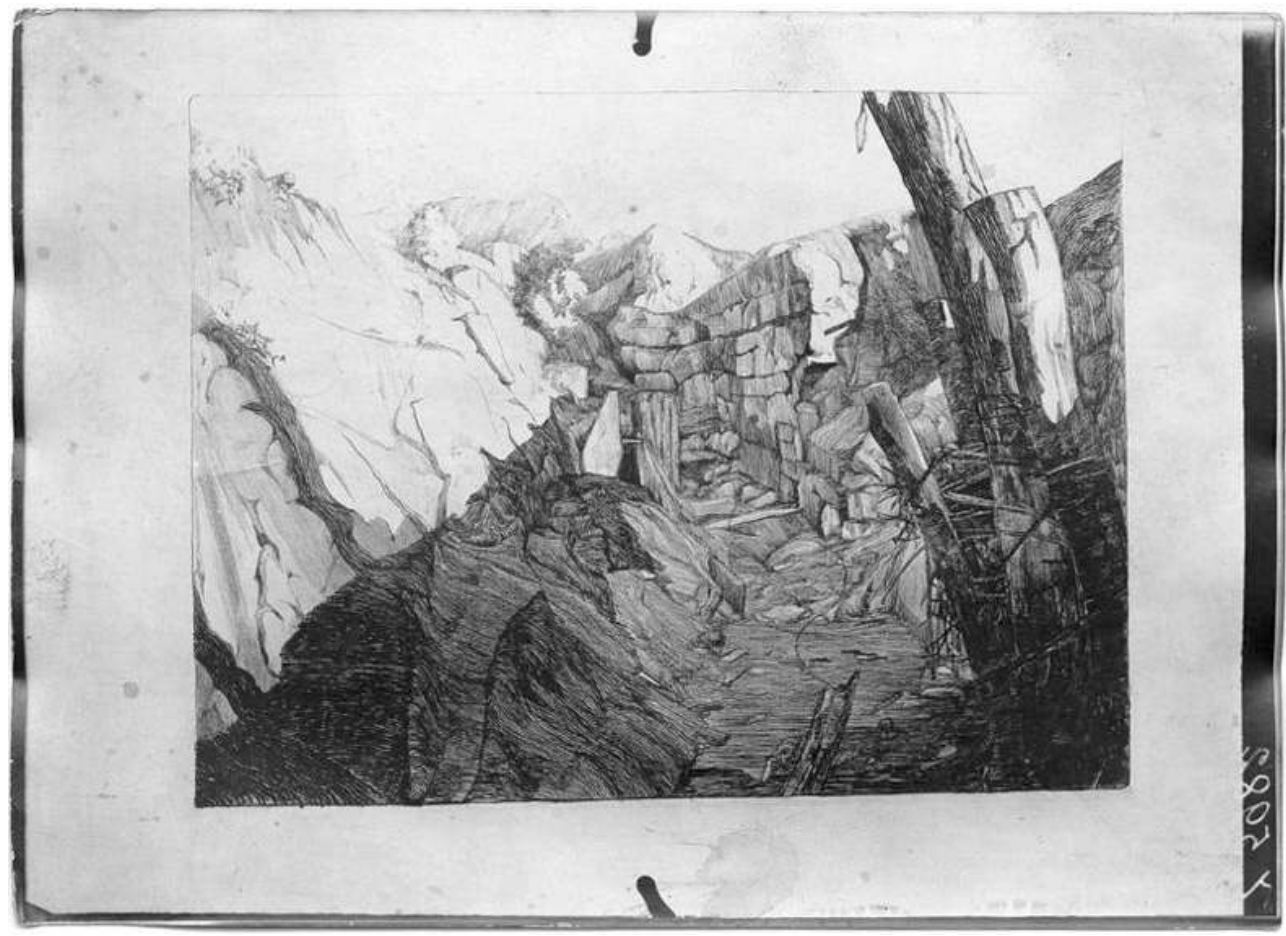

Tracy-le-Val (Oise), tranchée au cimetière, dessin d'André Ventre, juin 1917.

(c) Médiathèque de l'architecture et du patrimoine. 


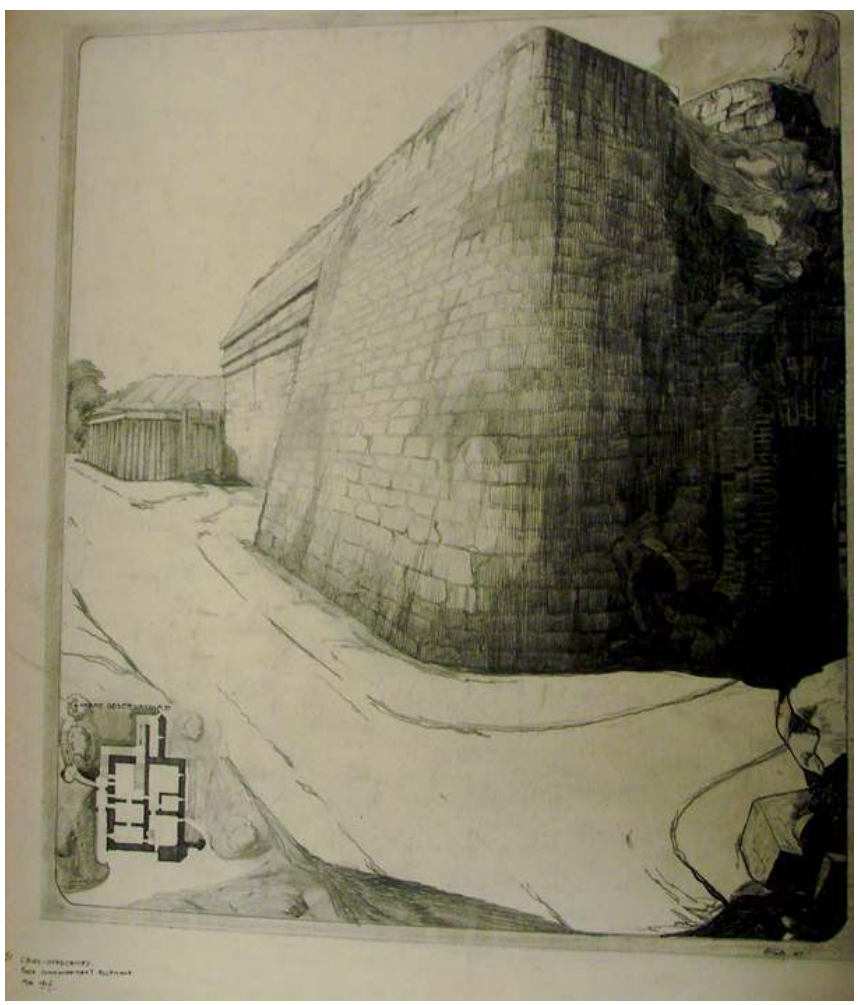

Chiry-Ourscamp (Oise), abri pour officiers allemands, dessin d'André Ventre, juin 1917 (non coté).

Phot. Viltart, Franck. (c) Médiathèque de l'architecture et du patrimoine.

À Dreslincourt, faute de temps certainement, André Ventre et le commandant Viel ne peuvent visiter les carrières occupées par les Allemands et notamment celle des Cinq Piliers. À la ferme d'Attiche, où les délégués notent que les installations commencent déjà à s'effondrer, aucun classement n'est envisagé. Les nombreuses maisons blindées dans le village de Thiescourt méritent simplement, selon eux, d'être photographiées. Notons que les deux hommes ne vont pas visiter la carrière du Chauffour, pourtant déjà connue pour son remarquable aménagement et ses sculptures monumentales ${ }^{20}$. Pour la butte-témoin du Plémont près de Lassigny, où les organisations allemandes sont très nombreuses, les délégués proposent le classement de l'ensemble du site. En contrebas, les ruines du château du Plessis-de Roye sont également proposées au classement. Les moindres stigmates de la guerre semblent les intéresser, comme le calvaire de Crapeaumesnil, avec son christ renversé et ses arbres alentour déchiquetés, qui pourra être conservé en l'état, selon eux. Un ensemble de cratères de mines près du village de Beuvraignes retient l'attention des deux hommes, qui signalent un cratère subsistant, qu'ils estiment à près de 200 mètres de circonférence pour 50 mètres de profondeur.

Les photographies prises lors de cette visite ainsi que les dessins d'André Ventre sont présentés à la séance de la commission le 22 juillet 1917, qui valide alors cette première liste de propositions de classement. Une seconde inspection est menée du 11 au 14 septembre 1917, dans une zone comprise entre Notre-Dame-de-Lorette et Roye. Intervenant en partie en secteur britannique, les délégués sont guidés par l'officierinterprète Bonnard, détaché de la Mission militaire française auprès des armées britanniques $^{21}$. Un rapport des deux délégués, daté du 15 octobre 1917 et intitulé « Région 
au Nord-Ouest d'Arras et région de la bataille de la Somme ", est présenté à la commission le 22 octobre $^{22}$, en présence de Bluzet, Paul Léon, Georges Famechon, le lieutenant-colonel Toutain et le maréchal des logis Dorange, secrétaire de la commission.

\section{Critères et conservation}

Il apparait très clairement à travers les premiers travaux de la commission qu'il ne s'agit pas seulement de procéder à l'inventaire des monuments ruinés, mais bien de prendre en considération un nouveau patrimoine avec ces vestiges produits par la guerre et notamment des éléments d'architecture militaire, des tranchées, des cratères de mines, des abris bétonnés, des postes d'observation, etc. Il est important de noter que pour la première fois, des propositions de classement sortent du cadre esthétique réservé aux monuments architecturaux pour y associer des villages en ruine ou des installations en béton.

Enthousiastes, les membres de la commission voient dans leur travail le moyen de garder « intacts, si cela est possible, les emplacements où se sont livrés des combats glorieux pour nos armées, dont le nom se retrouvera dans tous les documents écrits relatifs à la guerre $»^{23}$. Ils posent également clairement la vocation pédagogique de tels témoignages conservés et n'hésitent à faire appel à l'émotion que procure la vue des ruines :

Au point de vue éducatif, pour les générations futures, l'aspect d'un village démoli par l'artillerie ou par des explosions de mines, ou volontairement détruit par l'ennemi en retraite, sera autrement émotionnant et laissera un souvenir autrement frappant et durable que celui d'un poste de commandement ou d'un arbre observatoire ${ }^{24}$

André Ventre et le commandant Viel souhaitent attirer l'attention sur les destructions volontaires de la part des Allemands lors du retrait de mars 1917. Il importe selon eux de pouvoir témoigner des destructions volontaires de l'ennemi, parce que "l'émotion qui se dégage de ces destructions volontaires et systématiques opérées sans aucune utilité militaire est autrement intense que celle que produit la vue des villages à peu près disparus, au cours de la lutte ou des bombardements $»^{25}$. Les destructions volontaires opérées pour ralentir l'effort français lors du repli allemand seraient à leurs yeux bien plus fortes que les destructions dues aux combats. Il est frappant de voir ici que les choix proposés à la commission font intervenir un certain nombre de critères, tels que l'émotion, la pédagogie, le caractère édifiant des destructions ou même la nature de l'événement guerrier.

La reconstruction entre très vite au centre des débats de la commission des Vestiges et souvenirs de guerre. Le premier rapport à la commission commence par exposer le fait que les villages rencontrés sont totalement à terre et qu'il n'y plus aucun vestige à soumettre à un quelconque classement, "classer les ruines telles quelles sont, ce serait empêcher les villages de renaître » jugent les deux rapporteurs. À cause de la végétation qui recouvrira bientôt ces ruines, « l'émotion que fait naître l'aspect actuel de ces ruines ne subsisterait même pas " précisent-ils. André Ventre et le commandant Viel ajoutent que bien que les grands ensembles soient plus édifiants, « vouloir conserver dans leur état actuel de nombreuses organisation d'ensemble, ce serait empêcher la reconstruction des villages ou des grandes fermes sur leur emplacement primitif, ce serait priver l'agriculture de vastes terrains fertiles ». Ils proposent que seul un petit nombre de ces ensembles soient conservés en l'état et classés parmi les vestiges de guerre, soit par leur 
aspect historique, ou bien leur importance documentaire, ou encore parce que leur conservation ne nuirait pas à la reprise économique.

Comme dans le projet de 1915, André Ventre s'intéresse également au devenir des centres urbains mis à terre par les bombardements. Il visite la ville d'Arras complètement en ruines en 1917. Il propose d'y classer parmi les vestiges de guerre la Grand Place, la cathédrale, le cloître et le beffroi. La ville, avec ces monuments en ruine, servirait de décor pour appuyer le discours sur les désastres de la guerre, telle une pièce principale du martyrologue en cours de constitution.

Cependant, le souci de concilier conservation et impératifs de reconstruction demeure très présent au sein des travaux de la commission. Dans la Somme, près de Péronne, des vestiges très impressionnants de réseaux de tranchées près du Mont-Saint-Quentin ne sont pas retenus tandis qu'un observatoire bétonné est proposé au classement parce qu'il "pourrait être conservé sans gêner en quoi que ce soit la restauration de la propriété " ${ }^{26}$. À Pozières, un abri bétonné allemand à deux étages pourrait être également conservé dans les ruines de la sucrerie (fig. $\mathbf{n}^{\circ} \mathbf{7}$ ). En revanche, les énormes entonnoirs de mines de la Boisselle, témoins du déclenchement de l'offensive britannique du $1^{\text {er }}$ juillet 1916 sont, selon eux, « à conserver en l'état comme spécimen de la guerre des mines».

Figure 7

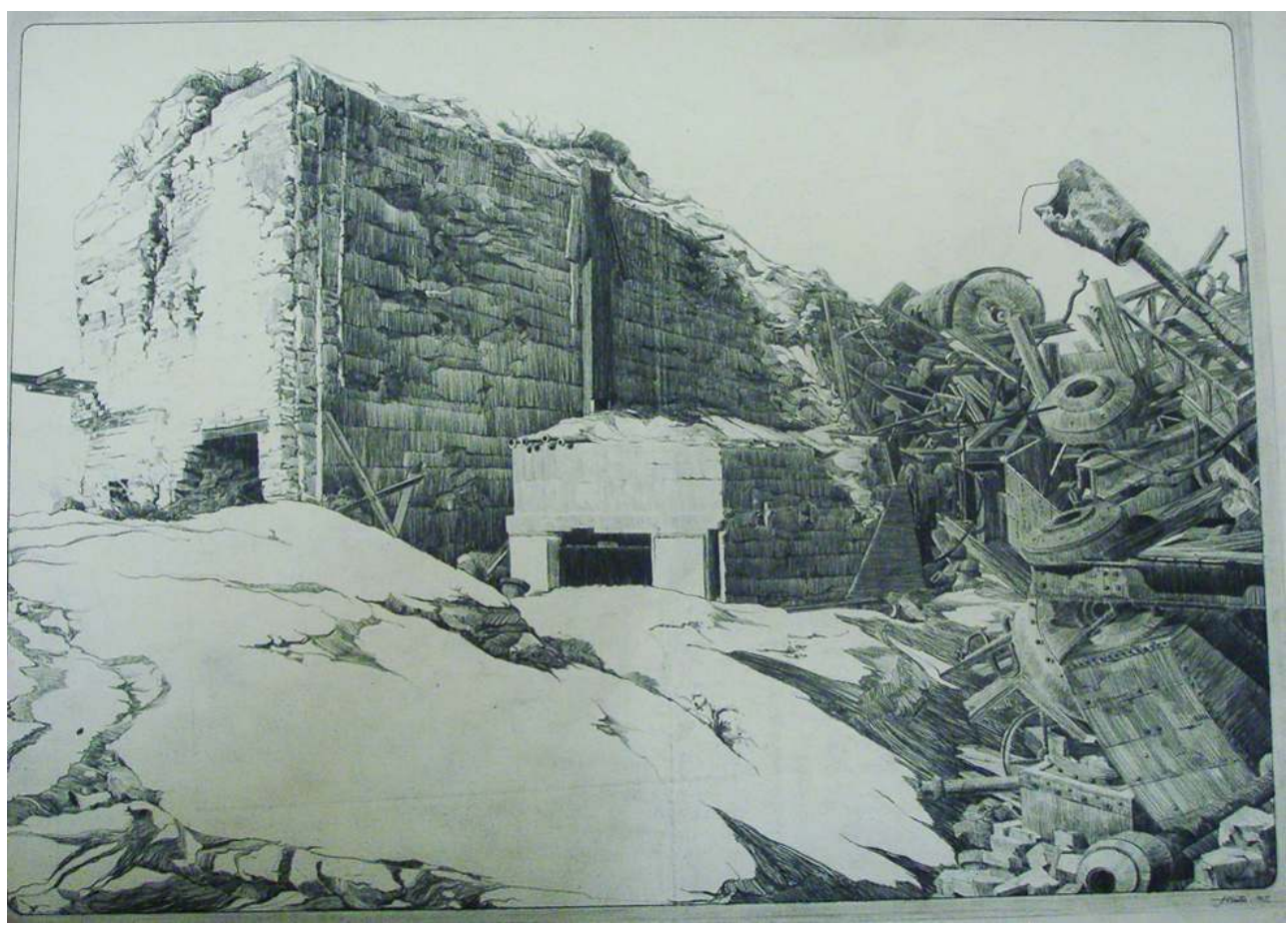

Pozières (Somme), abri bétonné allemand à deux étages, dessin d'André Ventre, septembre 1917 (non coté).

Phot. Viltart, Franck. (c) Médiathèque de l'architecture et du patrimoine.

La commission pointe aussi le problème de la conservation de ces vestiges, souvent faits de matériaux périssables. Dans l'attente de tout classement, l'armée confie à certaines unités du Génie la garde des premiers vestiges proposés par la commission en 1917 « jusqu'au jour où les circonstances permettront aux Beaux-Arts de prendre en charge les organisations classées ». En Picardie, le retour à l'offensive des Allemands en 1918 devait différer cet effort de préservation. Ces précautions, ainsi que les arguments avancés pour 
le classement des vestiges et souvenirs de guerre, montrent qu'avant même la fin des hostilités, ce processus de patrimonialisation correspondait à la nécessité d'entretenir une mémoire collective dont on pressentait l'importance une fois la guerre achevée.

\section{Une mémoire anticipée}

Le classement parmi les monuments historiques de vestiges ou de souvenirs de guerre permettait de faire accéder certains d'entre eux au rang de patrimoine national. La constitution d'un patrimoine à partir de vestiges d'une guerre qui n'était pas encore achevée démontre une simultanéité singulière et totalement nouvelle dans le domaine patrimonial.

La gestion de la mémoire s'affirme comme l'un des enjeux pour les acteurs principaux de la guerre. C'est pourquoi l'armée est particulièrement soucieuse de sa mise en scène par l'intermédiaire de cet effort de patrimonialisation. Étroitement encadrée par l'armée, la commission des Vestiges et souvenirs de guerre suit les recommandations des militaires. Le travail de la commission se place dans un récit historique de la guerre orchestré dans un premier temps par eux. Cependant, il est intéressant de voir qu'elle ne cherche pas à surdimensionner la part des vestiges français, mais bien ceux des plus remarquables. Les délégués se montrent particulièrement soucieux de présenter les traces des installations défensives allemandes qui s'avèrent très nombreuses dans la zone du front, notamment en Picardie. André Ventre signale toutefois que la liste fournie par le GQG s'est révélée insuffisante et qu'une étude méthodique des vestiges permettrait de découvrir des sites plus intéressants et souligne que l'observation des cartes militaires de canevas de tir pourrait être particulièrement utile. Pour cela, le secrétaire de la commission sert également de documentaliste à André Ventre, qui lui confie le rassemblement de tous les documents pouvant servir au classement des vestiges. Celui-ci rappelle que «la commission pourrait également faire appel utilement à la documentation du service des monuments et œuvres d'art de la zone des Armées (Conservation Évacuation) créée récemment par les soins du ministère de la Guerre et du sous-secrétaire d'État des BeauxArts $[\ldots] »^{27}$.

L'un des membres de la commission, Georges Famechon, propose également que soient édifiées des tables d'orientation sur les sites où le panorama est le plus intéressant. Le directeur de l'office national du Tourisme se déclare également prêt à préparer des brochures à partir des photos et des dessins rassemblés. La proposition qui n'est pas retenue par la commission fait cependant écho au projet éditorial mis en place au même moment par la société Michelin qui est en train d'éditer son premier guide bleu des champs de bataille en $1917^{28}$.

Pour favoriser la constitution d'un substrat mémoriel cohérent autour des vestiges de guerre, la commission dépasse sa mission initiale avec quelques propositions. André Ventre et le commandant Viel semblent avoir été frappés par l'installation précoce du souvenir britannique sur le champ de bataille. Leur visite dans la Somme en 1917, leur a ainsi permis d'observer l'édification de nombreux monuments ou simples croix par les Britanniques, en l'honneur de leurs régiments ou de leurs hommes tombés au combat. Bien que la commission n'ait pas d'attribution pour le faire, ses membres proposent tout de même d'envoyer une note au GQG afin qu'il envisage l'élévation de monuments commémoratifs provisoires propres aux unités françaises. Une proposition entendue au 
GQG, qui demande en 1917 de recenser les lieux des principales batailles de la guerre, à l'échelle des divisions françaises qui y ont participé. Une liste complète est établie en 1918, recensant plusieurs dizaines de sites sur lesquels pourraient être édifiés ces monuments provisoires, sur l'ensemble de la ligne de front ${ }^{29}$. N'ayant semble-t-il pas été informée par l'armée, notons que la commission n'a pas eu connaissance des très nombreux monuments commémoratifs allemands retrouvés dans la zone du retrait de 1917. La proposition d'installation de monuments commémoratifs provisoires français montre que la patrimonialisation des traces du conflit ne suffisait pas à sanctuariser le champ de bataille, qui devait pour cela être accompagné de nouveaux repères spatiaux identitaires.

Après les premiers rapports de la commission des Vestiges et souvenirs de guerre, on décide en 1918 de confier la suite des reconnaissances et la surveillance des sites au service de Protection des monuments historiques et œuvres d'art de la zone des armées. Tandis que la commission continue de valider des propositions de classement, on demande alors aux Beaux-Arts de préparer un nouveau projet de loi en vue du classement des « immeubles et les objets mobiliers dont la conservation présente un intérêt national au point de vue de l'histoire de la guerre ». Les critères du classement au titre des monuments historiques tels qu'énumérés dans la loi de 1913 n'étaient décidemment pas adaptés au projet de classement des vestiges de guerre. Un nouveau projet de loi est préparé par Albert Dalimier fin 1917, car les propositions de classement de la commission « ne pouvaient être ratifiées par arrêté, tant que des mesures législatives n'auraient pas étendu aux vestiges et souvenirs de guerre, les dispositions de la loi du 31 décembre $1913 »^{30}$. Pour la conservation de tous ces vestiges, le projet de 1917 propose qu'un droit de visite soit demandé, dont les sommes perçues pourraient être récoltées par les départements où se trouvent les biens classés, afin qu'ils entretiennent et gardent ces vestiges, interdisant par la même occasion à des propriétaires privés de tirer un quelconque profit des visites. Le premier article propose également que soient désormais soumis à la loi de 1913: "les immeubles et les objets mobiliers dont la conservation présente un intérêt national au point de vue de l'histoire de la guerre ».

À partir du texte de Dalimier, un nouveau projet de loi est présenté par le ministre de l'Instruction publique et des Beaux-Arts au nom du président de la République Alexandre Millerand, le 8 novembre $1920^{31}$. Puis, un nouveau projet est présenté par André Fribourg en 1921, avec cette fois-ci une liste en annexe de sites par département, qui, adopté par la Chambre le 9 février 1922, ouvre la voie à de nouveaux travaux de la commission des Vestiges et souvenirs de guerre.

Dans ces projets de classements, il importe de souligner le rôle que joua André Ventre. L'architecte continue de jouer un rôle central dans ceux-ci après-guerre, avec le transfert de la commission des Vestiges et souvenirs de guerre au sein des Monuments historiques en 1920. Il y poursuit inlassablement son travail d'inventaire dans chaque département, en collaboration avec les préfets et les architectes des Monuments historiques, afin de procéder à la protection, la surveillance et même à la restauration de certains vestiges. L'architecte trouve également l'occasion de mettre en œuvre ses propositions de monuments commémoratifs, en réalisant le monument de la Tranchée des Baïonnettes à Verdun en 1920, édifice également classé au titre des monuments historiques dès 1922. 


\section{NOTES}

1. - Voir à ce sujet AUDUC, Arlette. Quand les monuments construisaient la nation. Le service des monuments historiques de 1830 à 1940. Paris : La documentation française, 2008, p. 403-406.

2. - Médiathèque de l'Architecture et du Patrimoine (MAP), 80/3/5. Rapport fait au nom de la commission des Dommages de guerre chargée d'examiner le projet et les propositions de loi sur la réparation des dommages causés par les faits de la guerre, cité par AUDUC, Arlette, op.cit., p. 396.

3. - MAP, $80 / 11 / 59$.

4. - Jules-Luis Le Breton est originaire de Courrières dans le Nord ; socialiste proche de Jaurès, il devient sous le gouvernement Briand sous-secrétaire d'État aux inventions intéressant la défense nationale.

5. - MAP 90/03/36.

6. - Albert Dalimier est sous-secrétaire d'État en charge des Beaux-Arts du 14 juin 1914 à novembre 1917.

7. - WINTER, Jay. "Sites of Memory, Sites of Mourning”. The Great War in European Cultural History. Cambridge : Cambridge University Press, 1995, p. 78-116.

8. - LENIAUD, Jean-Michel. Les archipels du passé, le patrimoine et son histoire. Paris : Fayard, 2002, p. 213.

9. - MAP 90/03/37, lettre du Touring Club de France à Paul Léon, le 5 juin 1916.

10. - MAP 80/03/36, lettre de Paul Dalimier au garde des sceaux, ministre de la Justice.

11. - MAP 80/03/36, lettre du président de l'office national du Tourisme à Paul Léon.

12. - MAP 80/03/37, copie de la délibération du conseil général.

13. - MAP 80/3/37. Paul Léon est alors le directeur du service d'Architecture au sein du soussecrétariat aux Beaux-Arts (et à ce titre, est chargé des Monuments historiques), il publie deux ouvrages importants durant la guerre : Les monuments historiques, conservation, restauration. Paris : H. Laurens, 1917 ; et La renaissance des ruines, maisons, monuments. Paris : H. Laurens, 1918.

14. - MAP $80 / 3 / 37$.

15. - MAP $80 / 3 / 37$.

16. - Note du Grand Quartier général $N^{\circ} 6762$, datée du 8 mai 1917. Service historique de la Défense $16 \mathrm{~N} 1658$.

17. - Ces dessins sont aujourd'hui conservés à la Médiathèque de l'Architecture et du Patrimoine, fort de Saint-Cyr.

18. - MAP 80/3/37.

19. - Les carrières et les ruines de l'ancienne ferme de Confrécourt sont inscrites à l'inventaire des monuments historiques depuis le 28 juin 1990.

20. - Voir au sujet de ce site : HARDIER, Thierry. « La carrière du Chauffour et les traces rupestres de la Grande Guerre ». 1914-1918, l'Oise dans la Grande Guerre. Archives départementales de l'Oise, Beauvais, 2011.

21. - Voir sur cette seconde inspection : LONGEARD, Gwladys. «La lettre du 4 septembre 1917 relative au classement des vestiges de guerre dans la Somme ». Service historique de la Défense, département de l'armée de Terre, 17N305, Revue Historique des Armées, 242 / 2006 : 1916, les grandes batailles et la fin de la guerre européenne, p. 94-97.

22. - MAP 80/03/37.

23. - MAP 80/03/37, rapport de la commission des Vestiges et souvenirs de guerre, juillet 1917.

24. - MAP 80/03/37, rapport de la commission des Vestiges et souvenirs de guerre, juillet 1917. 
25. - MAP 80/03/37, rapport de la commission des Vestiges et souvenirs de guerre, juillet 1917. 26. - MAP 80/03/37, rapport de la commission des Vestiges et souvenirs de guerre, octobre 1917. 27. - MAP 80/03/37, rapport de la commission des Vestiges et souvenirs de guerre, octobre 1917. 28. - Voir DANCHIN, Emmanuelle. « Héroïsation des ruines et des combattants : la mise en place d'un tourisme de champ de bataille (1914-1921)». Dans AUZAS, Vincent, JEWSIEWICKI, Bogumil (dir.). Traumatisme collectif pour patrimoine. Regards sur un mouvement transnational, actes du colloque de Trois-Rivières du Québec des 7-8 mai 2007. Limoges : PUL, 2008, p. 237-260.

29. - SHD $16 \mathrm{~N} 1658$.

30. - MAP 80/03/36, Journal officiel, proposition de loi sur le classement des vestiges et souvenirs de guerre, 1920.

31. - MAP 80/03/36, Journal officiel, proposition de loi sur le classement des vestiges et souvenirs de guerre, 1920 .

\section{RÉSUMÉS}

La protection des vestiges de la Première Guerre mondiale relève en France d'un processus de patrimonialisation qui débute bien avant la fin de la guerre. Dès 1915, plusieurs projets voient le jour afin de conserver certaines ruines mais également des vestiges des combats, témoignages d'un conflit ravageur. En 1917 est constituée une commission des Vestiges et souvenirs de guerre, au sein de laquelle l'architecte en chef des Monuments historiques André Ventre œuvre à l'inventaire et à l'établissement des premières propositions de classement. À travers ce processus sans précédent, on assiste à une véritable anticipation de la mémoire du conflit relayée par un tourisme de guerre naissant.

The protection of the physical vestiges of the First World War is part of a heritage process which began well before the end of the war. As early as 1915, there were several projects for keeping certain ruined buildings and remains of particularly ravaged battlefields. A Commission des Vestiges et souvenirs de Guerre (War Memory and Relics Commission) was set up in 1917. As member of this commission, the Historic Monuments architect André Ventre, undertook an inventory and put forward the first proposals for sites worthy of statutory protection. This unprecedented operation may be seen as an anticipation of the memory of the conflict, encouraged by battlefield tourism which was already developing.

\section{AUTEUR}

\section{FRANCK VILTART}

Docteur en histoire, Mission Chemin des Dames fviltart@cg02.fr 